\title{
Panorama científico de intervenciones y formación profesional de entrenadores: una revisión sistemática
}

\section{Scientific panorama of coaches' interventions and professional learning: a systematic review}

\author{
Vinícius da Silva Musa, and Rafael Pombo Menezes* \\ Escola de Educação Física e Esporte de Ribeiräo Preto (EEFERP), Universidade de São Paulo (USP), (Brasil).
}

\begin{abstract}
The aim of this study was to identify the scientific scenario situation of the handball coaches. For this, a systematic review was performed with searches in different databases since 2000 in three specific themes: a) coaches intervention in performance; b) coaches intervention in psychology issues; and c) coaches profiles and formation. The analyzed papers highlighted the coaches professional learning, and the mainly investigated subjects were related to the coaches' interventions related to the technicaltactical contents, the feedbacks, the physical fitness, the leaderships ways and the relationship with players. It can be concluded that studies with coaches should be stimulated to assist them in the professional formation process, but greater attention should also be given to the youth sports, once that was found a preference for works involving adult teams.

Keywords: Bibliometry; Systematic review; Handball coaches.
\end{abstract}

Resumen: El objetivo de este estudio fue identificar el panorama científico con respecto a los estudios con entrenadores de balonmano. Para esto, se realizó una revisión sistemática con búsquedas en diferentes bases de datos desde el año 2000. El análisis de los artículos seleccionados permitió identificar tres temas: a) intervención de los entrenadores para el desempeño; b) intervención de entrenadores centrados en aspectos psicológicos; y c) perfil y formación de entrenadores. Los artículos analizados destacaron la formación de los entrenadores, teniendo como temas principales abordados por los investigadores las intervenciones de los entrenadores relacionadas con los contenidos técnico-tácticos, los comentarios, la preparación física, las formas de liderazgo y la relación con los jugadores. Se concluyó que los estudios con entrenadores deben ser estimulados para ayudarlos en el proceso de aprendizaje profesional, pero también se debe dar más énfasis a las categorías iniciales de entrenamiento deportivo, ya que se encontró preferencia por artículos que retratan la categoría de adultos.

Palabras-clave: Bibliometría; Revisión sistemática; Entrenador de balonmano.

\section{Introducción}

El balonmano es un deporte practicado internacionalmente que despierta el interés de académicos de diferentes áreas del conocimiento (Prieto, Gómez \& Sampaio, 2015), que buscan comprender los diferentes contextos y necesidades del deporte (Menezes, Marques \& Nunomura, 2015; Rivilla-García, Sillero, Grande, Sampedro \& Gómez, 2014).

Diferentes protagonistas influyen en los contextos de enseñanza y aprendizaje y/o entrenamiento de alto rendimiento en balonmano, y el entrenador toma una posición prominente en los procesos inherentes a ambos contextos. Históricamente, los estudios con entrenadores deportivos en Brasil han sido desarrollados por investigadores en el campo de la Pedagogía Deportiva (Marques, Nunomura \& Menezes, 2016), expresando preocupación por la enseńanza del deporte.

El entrenador deportivo desarrolla sus actividades profesionales en un entorno complejo, donde existe la interacción de diferentes agentes, en un proceso de formación que se consolida a lo largo de la carrera (Jones \& Thomas, 2015), con la

Dirección para correspondencia [Correspondence address]: Rafael Pombo Menezes. Universidade de São Paulo (USP), (Brasil).

E-mail: rafaelpombo@usp.br transmisión de valores durante las distintas prácticas profesionales (Cushion, Armour \& Jones, 2003). Los estudios que muestran el contexto de los entrenadores de balonmano son aún escasos, y el análisis de las producciones científicas permite identificar posibles tendencias en un campo particular de investigación (Aguilar, 2014). Por lo tanto, las revisiones sistemáticas son importantes para identificar el estado del arte de un tema determinado y guiar a los investigadores en su trabajo (Fernández-Ríos \& Buela-Casal, 2009).

Se pueden destacar dos revisiones sistemáticas del balonmano. En la primera, Aguilar (2014) realizó una encuesta sobre la producción de balonmano en artículos de revistas indexados en la Web of Science entre 1900 y 2014. El autor realizó un análisis bibliométrico de 366 artículos encontrados (origen de los autores, principales revistas, área de investigación, año de publicación e instituciones de autores). En la segunda, Prieto et al. (2015) buscaron en las bases de datos Web of Science (entre 1900 y 2012) y Medline (entre 1950 y 2012). Los autores analizaron en 373 artículos diferentes aspectos, como el año de publicación, el área de investigación, el tipo de documento, el idioma y las principales revistas.

Ambas revisiones (cuyo término de búsqueda fue "balon- 
mano") mostraron una tendencia creciente de artículos relacionados con el balonmano a lo largo de los años y señalaron un mayor interés en temas relacionados con la medicina deportiva, seguidos por las ciencias del deporte, el entrenamiento deportivo, la fisiología y la antropometría. Sin embargo, los estudios que incluyeron entrenadores, en particular sobre su educación y práctica profesional, no fueron objeto de estas revisiones.

Por lo tanto, dada la importancia del entrenador en el proceso de entrenamiento deportivo y la necesidad de guiar la posible investigación al respecto, el objetivo de este estudio fue realizar una revisión sistemática sobre entrenadores de balonmano para discutir las posibles fronteras del conocimiento sobre este tema en artículos más recientes.

\section{Método}

El diseño metodológico de esta revisión sistemática siguió los procedimientos sugeridos por Higgins y Green (2008), estableciendo: criterios de elegibilidad para los estudios, metodología explícita y reproducible, búsqueda sistemática, evaluación exhaustiva de los resultados del estudio y presentación sistemática de los estudios incluidos. Se basó en una pregunta inicial sobre una temática específica (“¿Cual es el panorama de los estudios con entrenadores de balonmano?”), lo que generó preguntas secundarias (“¿Cuáles son los temas más investigados sobre el entrenador de balonmano?”; “¿Cuáles son las brechas en este tema en la academia?), claramente formuladas que lo hizo posible "utilizar métodos sistemáticos y explícitos para identificar, seleccionar y evaluar críticamente la investigación relevante" (Galvão, Pansani \& Harrad, 2015, p.335), minimizar el sesgo y proporcionar resultados confiables (Higgins \& Green, 2008).

\subsection{Estrategias de búsqueda y criterios de inclusión}

Las directrices del PRISMA (Preferred Reporting Items for Systematic Reviews and Meta-Analyses) (Moher, Liberati, Tetzlaff \& Altman, 2009; Galvão et al., 2015) se utilizaron para la organización, la fiabilidad y la selección de los trabajos de este estudio. Las siguientes bases de datos fueron consultadas en enero / 2020: Scielo, Web of Science, EBSCOHost (especialmente considerando SPORTDiscus y Academic Search Premier), Lilacs, Redalyc, Scopus y Medline. Los términos de búsqueda utilizados en todas las bases de datos (en los cam- pos "título", "palabras clave" y "resumen") fueron "treinador" y "balonmano" (en portugués), "coach" y "handball” (en inglés) y "entrenador” y "balonmano" (en español).

La búsqueda en portugués se justifica por el intento de identificar y analizar investigaciones e información del contexto brasileńo y por ser el idioma fuente de los autores. La búsqueda en español se justifica por las premisas señaladas por Aguilar (2014), destacando a España como el país con el mayor número de artículos científicos publicados sobre balonmano, revelando su papel de liderazgo en el contexto académico. La búsqueda en inglés se justificó porque es el idioma universal de la ciencia (Volpato, 2011).

Para sistematizar la elección de los estudios en esta revisión y hacer factible la reproducibilidad de la búsqueda, seleccionamos trabajos que: (a) se indexaron en una de las bases de datos que se han presentado; (b) se publicaron en uno de los idiomas elegidos; (c) son originales y publicados en revistas desde 2000, con revisión doble ciego y textos completos disponibles en línea; y (d) el entrenador de balonmano figura como el principal protagonista.

En la selección fueron excluidos los artículos que no cumplieran con los criterios de inclusión (como artículos sin texto completo, artículos duplicados en la misma base de datos, resultado de la búsqueda en los tres idiomas, textos escritos en otros idiomas y libros, capítulos de libros, conferencias y anales de eventos). La última etapa consistió en la lectura de los títulos y resúmenes, verificando si el tema entrenador y balonmano figuraban como objeto central de cada estudio.

Después de encontrar 803 artículos en la búsqueda inicial se excluyeron 39 textos que se repitieron en las mismas bases de dados (encontrados en las búsquedas en los tres idiomas). Los 764 artículos seleccionados se movieron a una tabla, ordenados por fecha y nombre del autor, lo que permitió excluir artículos que se repitieron en diferentes bases (porque hay revistas indexadas en varias bases de datos) y/o cuyos títulos y resúmenes no contemplaban los criterios de inclusión. En esta etapa, se excluyeron 708 artículos. Por lo tanto, fueran incluidos 56 artículos para el análisis.

Los artículos seleccionados fueron clasificados en tres categorías: estudios de intervención de los entrenadores con respecto al rendimiento de los jugadores; estudios sobre la intervención de los entrenadores sobre aspectos psicológicos; y investigaciones sobre el perfil y el entrenamiento de los entrenadores. La Figura 1 muestra todos los pasos del diagrama de flujo para la selección de artículos. 


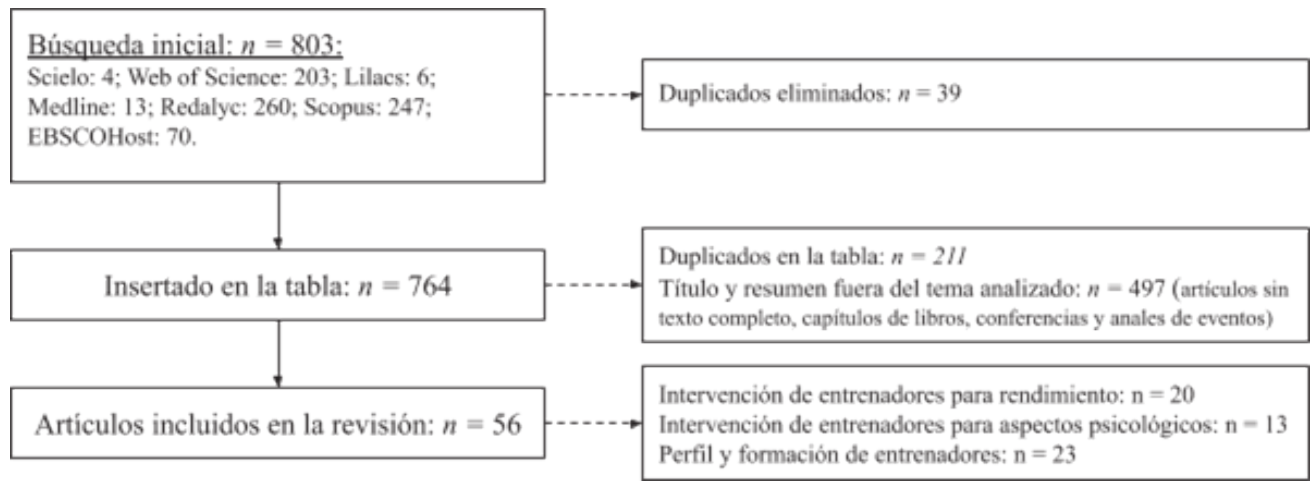

Figura 1. Diagrama de flujo de selección de artículos.

\subsection{Fiabilidad y análisis de datos}

Para no restringir el análisis de los datos a un punto de vista, la selección de artículos y su interpretación fueron realizados por los dos autores de este estudio, y debatidos con un grupo de estudiantes en el área, llegando así a las conclusiones presentadas. En consecuencia, para una interpretación más transparente de los estudios cualitativos, Tong, Flemming, McInnes, Oliver y Craig (2012) sugieren una exposición ge- neral de los resultados para una mejor comprensión del panorama, seguido de una discusión de los contenidos específicos.

\section{Resultados}

Esta sección presenta el resumen general de las publicaciones y los contenidos relacionados con los trabajos encontrados. La Figura 2 presenta el número de publicaciones en cada año desde 2005 (fecha de la primera aparición).

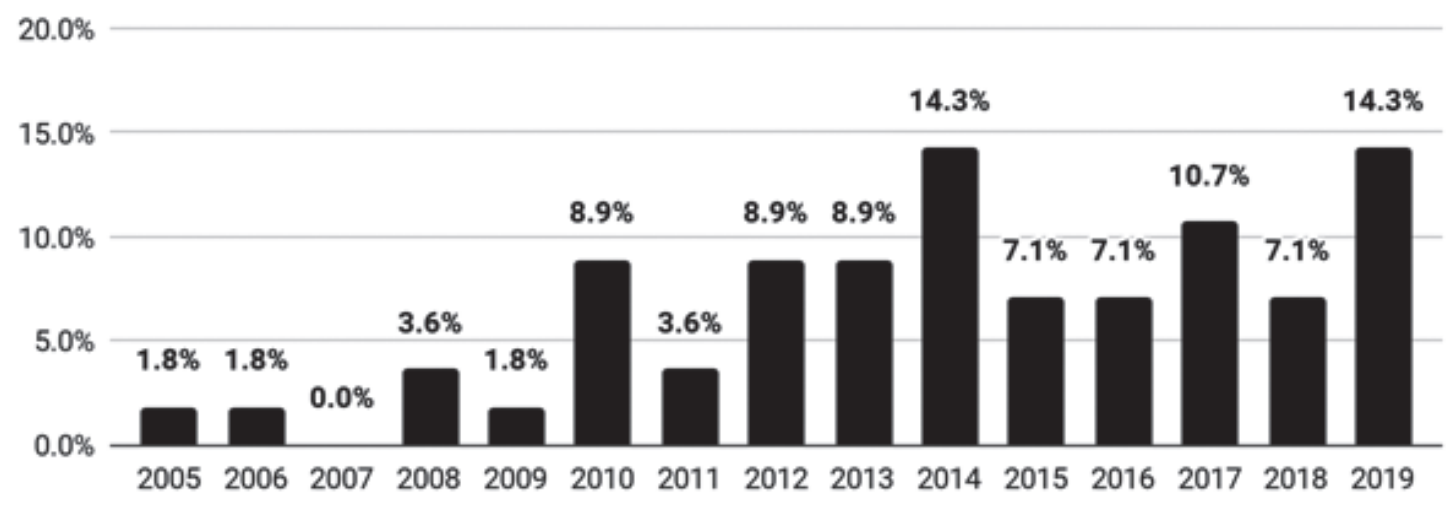

Figura 2. Porcentaje de artículos publicados por año.

Se observó un mayor número de publicaciones en 2014, 2019 (14.3\% en cada) y 2017 (10.7\%), y entre estos períodos hubo oscilaciones hacia abajo. El mismo comportamiento se observó en el período anterior a 2014. Aun así, se puede observar un creciente interés en el tema.

La Figura 3 muestra los porcentajes de artículos según el número de autores, que varió de uno a seis.

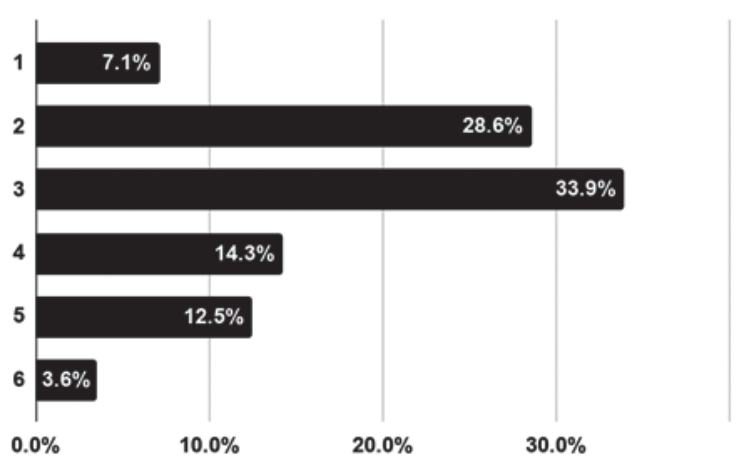

Figura 3. Porcentaje de autores por artículo. 
Los trabajos que abordaron el tema estudiado fueron escritos con mayor frecuencia por tres o dos autores $33.9 \% \mathrm{y}$ 28.6, respectivamente). Artículos con uno y seis autores fueran menos frecuentes, que revela la participación de pequeños grupos en publicaciones.

Otro dato analizado fue el número promedio de autores por artículo y por ańo, presentados en la Figura 4.

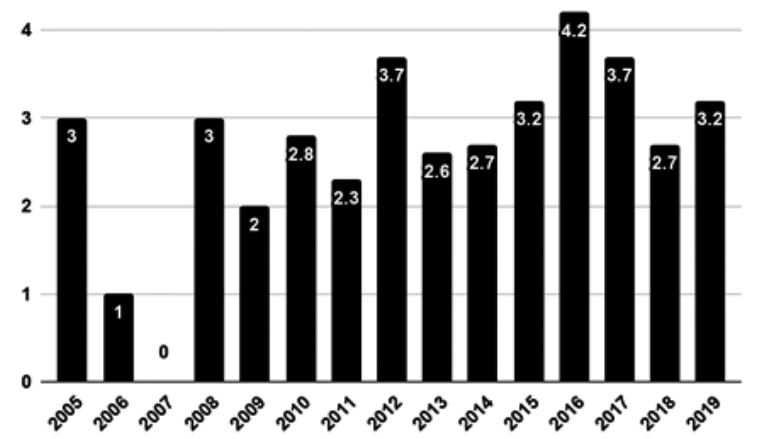

Figura 4. Número promedio de autores por artículo por año.

Los datos revelaron que los promedios más altos de autores por artículo se registraron en 2016, 2012 y 2016 (4.2 autores/artículo, 3.7 autores/artículo y 3.7 autores/artículo, respectivamente). Como se muestra en relación con el número de publicaciones por año (Figura 2), el comportamiento del número promedio de autores por artículo fue irregular (períodos alternos de aumento y disminución).

La Figura 5 muestra los países con mayor frecuencia de trabajo (Institución a la que está vinculado el primer autor).

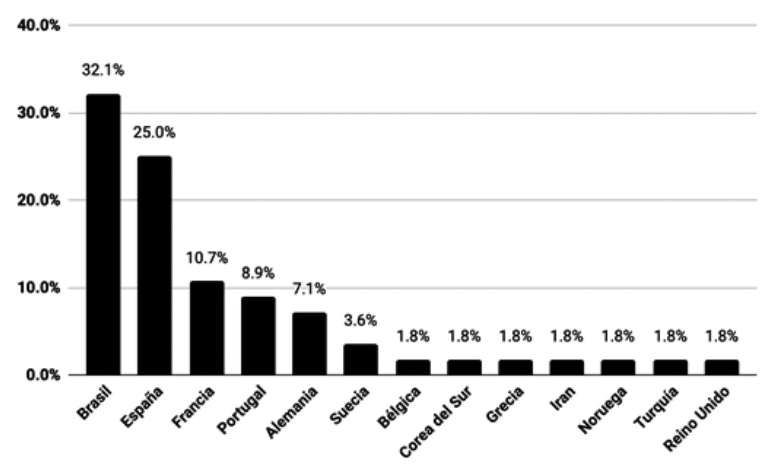

Figura 5. Porcentaje de publicaciones por país.

Aunque Brasil es el país con el mayor porcentaje de publicaciones $(32.1 \%)$, es posible identificar la predominancia de países europeos (64.3\% de las publicaciones). De manera complementaria, la identificación del idioma de los artículos está presentada en la Figura 6.

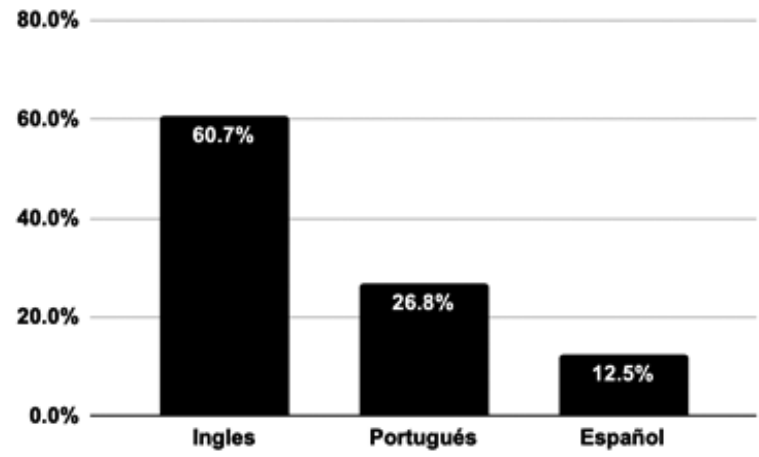

Figura 6. Número de publicaciones por idioma.

Se comprueba la predominancia del inglés $(60.7 \%)$ cuando comparado al portugués y al español, que revela el inglés como el idioma principal de los artículos sobre este tema.

Para una mejor comprensión y discusión de los trabajos analizados, estos fueron clasificados en categorías de acuerdo con sus objetos de investigaciones. En la categoría "Intervención de los entrenadores para rendimiento" (Figura 7) se encontró artículos que hay investigado sobre las cuestiones técnico-tácticas del juego y/o de la preparación física. Fue posible identificar el predominio de investigaciones con diseño cualitativo (40\% de los estudios en esta categoría), que utilizó las entrevistas para la recolección de datos y que enfatizó los aspectos técnico-tácticos como su principal objeto de investigación.

Los trabajos asignados en la categoría "Intervención de los entrenadores para aspectos psicológicos" (Figura 7), abordaron temas como las formas de relación con los equipos, la motivación de los jugadores debido a la forma de liderazgo y las diferentes estrategias de retroalimentación. Como en la categoría anterior, fue posible identificar que los autores optaron principalmente por enfoques cualitativos $(69 \%$ de los estudios en esta categoría) y el uso de entrevistas para investigar el comportamiento de los entrenadores con sus jugadores y equipos (38\% de los estudios en esta categoría).

Por fin, la tercera categoría "Perfil y formación de entrenadores" presenta los trabajos que investigaron las necesidades de los entrenadores en su formación profesional y las posibilidades para mejorar este proceso. En esta categoría hubo predominio de los enfoques cuantitativos (61\% de los estudios en esta categoría), en el que los cuestionarios fueran utilizados con mayor frecuencia para la recolección de datos y el tema más investigado fue la experiencia del entrenador.

La Figura 7 presenta un resumen de los estudios seleccionados, con énfasis en los porcentajes que se refieren a los tipos de investigaciones más frecuentes (cualitativa, cuantitativa o mixta), los métodos más utilizados para recopilar los datos (entrevistas, cuestionarios, observaciones, entre otros) y en los principales objetos de investigación. 


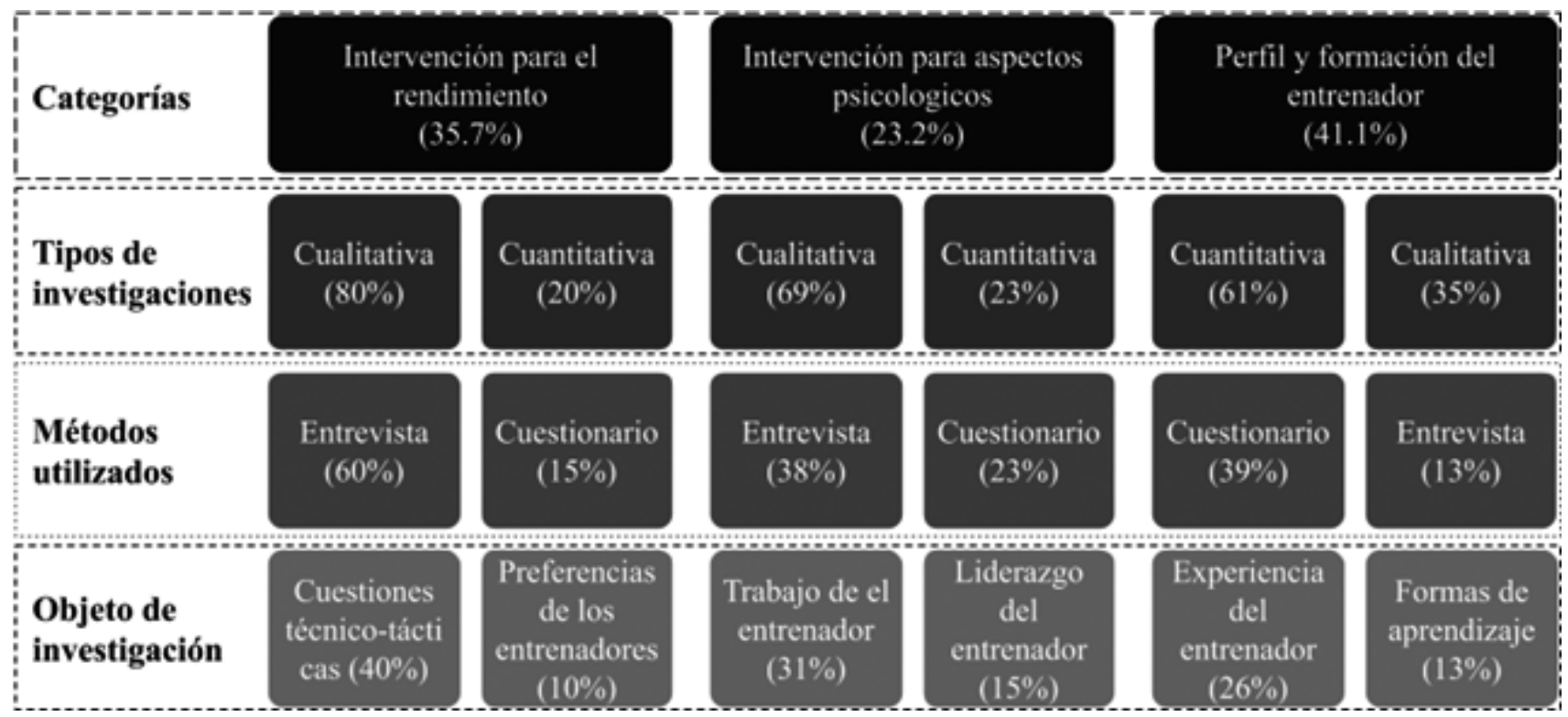

Figura 7. Diseños metodológicos más utilizados en cada categoría.

Al analizar los artículos de la categoría "Intervención de los entrenadores para rendimiento" (tabla 1) se encontró que $40.0 \%$ de los estudios seleccionados han abordado las opiniones de los entrenadores sobre los elementos técnico-tácticos más relevantes (Menezes \& Reis, 2014; Menezes, et al., 2015; Menezes \& Reis, 2017a, 2017b, 2017c; Krahenbühl, Menezes \& Leonardo, 2019; Modolo \& Menezes, 2019) y los más observados por ellos en el juego (Menezes; Morato \& Reis, 2015). Treinta y cinco por ciento de los trabajos analizados investigaron los procedimientos pedagógicos adoptados en diferentes etapas de la formación del jugador (Menezes, Marques \& Morato 2016; Menezes \& Reis, 2017c; Menezes, Ramos, Marques \& Nunomura, 2018; Dallegrave, Mendes \& Nascimento, 2018; Menezes, 2019) y el proceso de iniciación deportiva (Krahenbühl, Galatti, Scaglia \& Leonardo, 2019; Santos \& Menezes, 2019).

También se identificó, en la tabla 1 , que de los estudios que entrevistaron a entrenadores $(n=13), 53.9 \%$ se refieren al contexto del balonmano brasileño (entrenadores con participación en equipos nacionales o que trabajan en equipos municipales) y $46.1 \%$ al contexto de otros países

Otros estudios relacionados con la "Intervención de los entrenadores para rendimiento" (Tabla 1) (Meeus, Serpa \& De Cuyper, 2010; Rivilla-García et al., 2014), además del enfoque en la acción positiva de la retroalimentación positiva como una mejor forma de optimizar el rendimiento del jugador (Hoigaard, De Cuyper, Fransen, Boen \& Peter, 2015). Los enfoques cuantitativos encontrados en la tabla 1 también utilizaron cuestionarios con diversos temas, como el uso de ejercicios concéntricos y pliométricos en la preparación física del balonmano (Debanne \& Laffaye, 2013).

La tabla 1 presenta, en orden cronológico descendente, todos los artículos sobre el tema "Intervención de entrenadores para rendimiento" indicando los autores, el tipo de investigación, los métodos utilizados, las variables observadas y los participantes.

Tabla 1. Artículos que investigaron la intervención de los entrenadores para rendimiento.

\begin{tabular}{ccccc}
\hline Autores & Tipo & Método & Variable & Participantes \\
\hline Modolo y Menezes (2019) & Cualitativo & Entrevista & Opinión sobre técnica y táctica & $\begin{array}{c}19 \text { entrenadores de categoría } \\
\text { sub-16 }\end{array}$ \\
\hline Santos y Menezes (2019) & Cualitativo & Entrevista & $\begin{array}{c}\text { Opinión sobre la especialización en las } \\
\text { etapas de formación }\end{array}$ & 4 entrenadores (nacional) \\
\hline $\begin{array}{c}\text { Krahenbühl, Menezes y Leo- } \\
\text { nardo (2019) }\end{array}$ & Cualitativo & Entrevista & Opinión sobre técnica y táctica & 4 entrenadores (nacional) \\
\hline $\begin{array}{c}\text { Krahenbühl, Galatti, Scaglia y } \\
\text { Leonardo (2019) }\end{array}$ & Cualitativo & Entrevista & Opinión sobre la formación en el \\
balonmano & 4 entrenadores (nacional) \\
\hline $\begin{array}{c}\text { Dallegrave, Mendes y Nasci- } \\
\text { mento (2018) }\end{array}$ & Cuantitativo & Filmación & Preferencias de los entrenadores & 2 entrenadores y 27 jugadores \\
\hline
\end{tabular}




\begin{tabular}{|c|c|c|c|c|}
\hline Autores & Tipo & Método & Variable & Participantes \\
\hline Menezes (2018) & Cualitativo & Entrevista & $\begin{array}{l}\text { Opinión sobre lo ensino del balon- } \\
\text { mano }\end{array}$ & 6 entrenadores (nacional) \\
\hline $\begin{array}{c}\text { Menezes, Ramos, Marques y } \\
\text { Nunomura (2018) }\end{array}$ & Cualitativo & Entrevista & $\begin{array}{l}\text { Opinión sobre lo ensino del balon- } \\
\text { mano }\end{array}$ & 6 entrenadores (nacional) \\
\hline Menezes y Reis (2017b) & Cualitativo & Entrevista & Opinión sobre técnica y táctica & 4 entrenadores (nacional) \\
\hline Menezes y Reis (2017a) & Cualitativo & Entrevista & Opinión sobre técnica y táctica & 4 entrenadores (nacional) \\
\hline Menezes y Reis (2017c) & Cualitativo & Entrevista & Opinión sobre técnica y táctica & 4 entrenadores (nacional) \\
\hline $\begin{array}{l}\text { Menezes, Marques y Morato } \\
\text { (2016) }\end{array}$ & Cualitativo & Entrevista & Opinión sobre técnica y táctica & 4 entrenadores (nacional) \\
\hline $\begin{array}{c}\text { Menezes, Marques, et al. } \\
\text { (2015) }\end{array}$ & Cualitativo & Entrevista & Métodos de enseńanza & 6 entrenadores (regional) \\
\hline $\begin{array}{l}\text { Hoigaard, De Cuyper, Fransen, } \\
\text { Boen y Peter (2015) }\end{array}$ & Cuantitativo & Cuestionario & $\begin{array}{c}\text { Percepción del comportamiento del } \\
\text { entrenador }\end{array}$ & 113 jugadoras adultas \\
\hline Menezes, Morato y Reis (2015) & Cualitativo & Entrevista & Opinión sobre técnica y táctica & 4 entrenadores (nacional) \\
\hline Rivilla-García et al. (2014) & Cuantitativo & $\begin{array}{l}\text { Cuestionario } \\
\text { y prueba de } \\
\text { percepción }\end{array}$ & $\begin{array}{l}\text { Uso de las TIC y motivación de los } \\
\text { jugadores }\end{array}$ & 71 jugadores juveniles \\
\hline Menezes y Reis (2014) & Cualitativo & Entrevista & Opinión sobre técnica y táctica & 4 entrenadores (nacional) \\
\hline Debanne y Laffaye (2013) & Cuantitativo & Cuestionario & $\begin{array}{l}\text { Opinión sobre el aumento de la veloci- } \\
\text { dad del brazo de lanzamiento }\end{array}$ & 28 entrenadores \\
\hline Hallajy, Janani y Fallah (2011) & Cualitativo & Cuestionario & Efecto del liderazgo & 218 jugadores profesionales \\
\hline $\begin{array}{l}\text { Molina, Godoy, Gozalo y } \\
\text { Lorenzo (2010) }\end{array}$ & Cualitativo & Cuestionario & Planificación y decisión & $\begin{array}{l}334 \text { entrenadores con certifica- } \\
\text { ción federativa }\end{array}$ \\
\hline $\begin{array}{l}\text { Meeus, Serpa y De Cuyper } \\
\text { (2010) }\end{array}$ & Cualitativo & $\begin{array}{l}\text { Cuestionario } \\
\text { e grabación } \\
\text { de partidos }\end{array}$ & Efectos de feedback de video & 93 jugadores e 9 entrenadores \\
\hline
\end{tabular}

En la categoría "Intervención del entrenador centradas en aspectos psicológicos" (tabla 2) fue posible identificar un resaltado para la influencia de los aspectos psicológicos en el comportamiento, la cognición y la confianza del jugador (Moesch \& Apitzsch, 2012).

En esta categoría $53.8 \%$ de los estudios han intentado comprender la planificación del trabajo de los entrenadores a partir de sus opiniones (Debanne \& Fontayne, 2009; Molina, Ibáñez, Gozalo \& Lorenzo, 2010; Sonoo, Hoshino \& Vieira, 2008) o sus jugadores (Guzmán et al., 2005; Sonoo et al., 2008), además de la importancia de los objetivos de rendimiento en la mejora del equipo y la creación de confianza por parte de los jugadores (Guzmán et al., 2005). Sonoo et al. (2008) señalaron que las mujeres necesitan más apoyo social y los hombres necesitan información relacionada con las reglas y los problemas del juego para lograr el rendimiento.

La categoría "Intervención de los entrenadores para aspectos psicológicos" (tabla 2) presentó un estudio de caso con un entrenador sugirió que la comunicación y la relación con los jugadores deberían ser respetuosas, permitiéndoles participar en la toma de decisiones del equipo (Annerstedt \& Lindgren, 2014). También se abordaron las formas de liderazgo y relación de entrenadores con jugadores (Hallajy, Janani \& Fallah, 2011; Gomes \& Paiva, 2010), cuyos cuestionarios revelaron la prevalencia de resultados positivos cuando el entrenador (equipo portugués) utilizó el liderazgo transformacional en la convivencia con sus atletas (Gomes \& Paiva, 2010).

De los trabajos centrados en los aspectos psicológicos (tabla 2), $23.1 \%$ analizaron cualitativamente el rendimiento del entrenador utilizando la grabación de voz durante el entrenamiento y los juegos. Debanne et al. (2014) reforzaron la idea de respeto y buena comunicación para promover una mayor asistencia a los jugadores durante todo el partido. Al estudiar la relación del entrenador con sus jugadores, Bormann, Schulte-Coerne, Diebig y Rowold (2016) descubrieron que su desempeño individual puede mejorar o empeorar dependiendo de cómo se los guíe. Por lo tanto, se sugiere el uso del liderazgo transformacional y transaccional en la realización del trabajo, ya que pueden interferir con el comportamiento del individuo en las tareas diarias (Lee, Kim \& Kang, 2013).

Todavía en los artículos de esta categoría (tabla 2) se notó que la preparación psicológica ha demostrado la importancia del entrenador en la motivación en lo proceso de entrenamiento deportivo (Gómez-López, Manzano-Sánchez, Merino-Barreno \& Valero-Valenzuela, 2019; Silva, Barreiro \& Fonseca, 2019), que la orientación de la tarea es más motivadora para los jugadores que la orientación del resultado (Granero-Gallegos et al., 2017), y la importancia de entrenar 
a su equipo con los jugadores (Konig, 2013). En la tabla 2 entrenadores para aspectos psicológicos". se presentan los artículos sobre el tema "Intervención de los

Tabla 2. Artículos que investigaron la intervención del entrenador centrada en aspectos psicológicos.

\begin{tabular}{|c|c|c|c|c|}
\hline Autores & Tipo & Método & Variable & Participantes \\
\hline $\begin{array}{c}\text { Gómez-López, Manzano-Sán- } \\
\text { chez, Merino-Barreno y Valero- } \\
\text { Valenzuela (2019) }\end{array}$ & Cualitativo & Cuestionario & Motivación de los jugadores & $\begin{array}{c}444 \text { jugadores (16 y } 17 \\
\text { años) }\end{array}$ \\
\hline Silva, Barreiro y Fonseca (2019) & Cualitativo & Entrevista & $\begin{array}{l}\text { Opinión de los jugadores } \\
\text { sobre los entrenadores }\end{array}$ & $\begin{array}{c}14 \text { jugadores de distintos } \\
\text { niveles }\end{array}$ \\
\hline Granero-Gallegos et al. (2017) & Cuantitativo & Cuestionario & Motivación de los jugadores & 159 jugadores jóvenes \\
\hline $\begin{array}{l}\text { Bormann, Schulte-Coerne, Die- } \\
\text { big y Rowold (2016) }\end{array}$ & Cuantitativo & Cuestionario & $\begin{array}{l}\text { Diferentes formas de lide- } \\
\text { razgo }\end{array}$ & $\begin{array}{c}336 \text { jugadores adultos } / 30 \\
\text { entrenadores (regional) }\end{array}$ \\
\hline $\begin{array}{l}\text { Debanne, Fontayne y Bourbous- } \\
\text { son (2014) }\end{array}$ & Cualitativo & Grabación de voz & $\begin{array}{l}\text { Informaciones de los entrena- } \\
\text { dores en los partidos }\end{array}$ & $\begin{array}{c}3 \text { entrenadores profesiona- } \\
\text { les } / 15 \text { partidos }\end{array}$ \\
\hline Annerstedt y Lindgren (2014) & Cualitativo & Entrevista & $\begin{array}{c}\text { Estrategias y experiencias } \\
\text { exitosas }\end{array}$ & 1 entrenador \\
\hline Lee, Kim y Kang (2013) & Cuantitativo & Cuestionario & $\begin{array}{l}\text { Comportamiento de lide- } \\
\text { razgo }\end{array}$ & 73 jugadores profesionales \\
\hline König (2013) & $\begin{array}{l}\text { Cualitativo y } \\
\text { cuantitativo }\end{array}$ & $\begin{array}{l}\text { Entrevista y cues- } \\
\text { tionario } \\
\end{array}$ & Liderazgo del entrenador & 10 entrenadores \\
\hline Moesch y Apitzsch (2012) & Cualitativo & Entrevista & Momentum de los partidos & 9 entrenadores \\
\hline Gomes y Paiva (2010) & Cualitativo & Cuestionario & $\begin{array}{l}\text { Percepción del tipo de } \\
\text { liderazgo }\end{array}$ & 91 jugadores (13 a 42 años) \\
\hline Debanne y Fontayne (2009) & Cualitativo & $\begin{array}{l}\text { Entrevista y recuer- } \\
\text { do estimulado }\end{array}$ & Gestión cognitiva & 1 entrenador \\
\hline Sonoo, Hoshino y Vieira (2008) & Cualitativo & Entrevista & Liderazgo del entrenador & $\begin{array}{l}218 \text { jugadores juveniles y } \\
\text { adultos } / 20 \text { entrenadores }\end{array}$ \\
\hline $\begin{array}{l}\text { Guzmán, Garcia Ferriol y Gime- } \\
\text { no (2005) }\end{array}$ & Cualitativo & Entrevista & $\begin{array}{l}\text { Percepción de competencia en } \\
\text { el enfoque del entrenador }\end{array}$ & 72 jugadoras infantiles \\
\hline
\end{tabular}

En estudios relacionados con la categoría "Perfil y formación del entrenador" (tabla 3) se observó que en 30.4\% se señaló que las experiencias como entrenador y atleta en el deporte influyen en la eficiencia del trabajo (Meletakos, Bayios, Hatziharistos \& Psychountaki, 2013), en el aprendizaje (Molina, Godoy, Calvo, Saiz \& Alonso, 2012) y en el perfil del entrenador (Gómez-López, Martínez \& Ruiz-Sánchez, 2018); que la personalidad puede influir en la forma de liderazgo (Gama et al., 2019) y que las relaciones con otros entrenadores (Modolo, Madeira, Santos, D’Almeida \& Menezes, 2017; Musa, Modolo, Barreira, Tsuji \& Menezes, 2017), así como la influencia de los mentores (Cunha, Estriga \& Batista, 2014), son importantes en la formación profesional.

Trece por ciento de los estudios relacionados a formación y el perfil de los entrenadores (tabla 3) utilizaron la observación de juegos, grabación de voz y pruebas de computadora, que fueron analizados cuantitativamente por los investigadores, y señalaron una posible interferencia del tiempo de experiencia profesional del entrenador en su capacidad para tomar decisiones (Fischer et al., 2016; Hagemann, Strauss \& Büsch, 2008). También con las grabaciones de voz de entrenadores,
Luján et al. (2014) mostraron cómo la experiencia del entrenador y la experiencia deportiva pueden interferir con su motivación durante la práctica profesional.

Cuando los entrenadores han sido cuestionados acerca de la capacitación académica (tabla 3), los más experimentados informaron que los contenidos presentados en la capacitación inicial eran insuficientes (Feu Molina, Ibáñez Godoy \& Gozalo Delgado, 2010a), lo que sugiere una mayor atención al dominio de los contenidos específicos y a la influencia social en la elaboración de currículos académicos (Mesquita, Borges, Rosado \& Batista, 2012). Además, los entrenadores experimentados destacaron la relevancia del aprendizaje sobre planificación, cuestiones sociales, orientación colectiva innovadora y orientada a la tecnología (Feu Molina, Ibáńez Godoy \& Gozalo Delgado, 2010b; Mesquita, Borges, Rosado \& De Souza, 2011) y un estudio etnográfico demostró la necesidad de entender el desempeño del entrenador como algo amplio y derivado de la vida cotidiana (Jones \& Hemmestad, 2019).

De los artículos seleccionados en esta categoría (tabla 3), $13.0 \%$ compara el desempeño del entrenador con su grado de capacitación han demostrado que la capacitación académica 
puede brindar mayor competencia para ellos y los jugadores (Mesquita et al., 2012), así como aumentar el nivel de motivación de los jugadores (Pulido, Sanchez-Oliva, Sanchez-Miguel, Leo \& Garcia-Calvo, 2016). También se encontraron diferencias en la percepción de los jugadores de su relación con el entrenador según el nivel educativo (Çelik \& Yalçinkaya, 2015).

Debanne et al. (2014) revelaron que los entrenadores adoptan diferentes estrategias en detrimento de las diferentes situaciones presentadas por el juego, que son más defensivas en el segundo tiempo de los partidos y se centran en la preparación física y mental cuando se enfrentan a equipos de niveles de juego más cercanos (Debanne \& Fontayne, 2012). También se analizaron los comportamientos verbales de los entrenadores durante los juegos, de lo que se concluyó que pueden ayudar con mayor calidad a los jugadores en situaciones donde el equipo está ganando los partidos, es decir, con menos presión (Calpe-Gómez et al., 2013) y ofrecieron comentarios más positivos en momentos en que la acción fue exitosa (Lujan Guzmán \& Calpe-Gómez, 2012). Además, buscamos entender cómo los entrenadores se comunican con los árbitros durante los partidos (Debanne, 2014). La tabla 3 presenta todos los artículos que abordaron el tema "Perfil y formación del entrenador".

Tabla 3. Artículos que investigaron el perfil y la formación de entrenadores.

\begin{tabular}{|c|c|c|c|c|}
\hline Autores & Tipo & Método & Variable & Participantes \\
\hline $\begin{array}{l}\text { Gama, Nunes, Castro, Souza, } \\
\text { Junior y Vale (2019) }\end{array}$ & Cuantitativo & Cuestionario & Perfil de los entrenadores & 31 entrenadores (escuelas) \\
\hline Jones y Hemmestad (2019) & Cualitativo & Estudio Etnográfico & Actuación de el entrenador & $\begin{array}{l}\text { Equipo nacional noruego } \\
\text { feminino }\end{array}$ \\
\hline $\begin{array}{c}\text { Gómez-López, Martínez y } \\
\text { Ruiz-Sánchez (2018) }\end{array}$ & Cuantitativo & Cuestionario & Perfil de los entrenadores & $\begin{array}{c}76 \text { entrenadores (16 a } 57 \\
\text { ańos) }\end{array}$ \\
\hline $\begin{array}{c}\text { Musa, Modolo, Barreira, Tsuji } \\
\text { y Menezes (2017) }\end{array}$ & Cualitativo & Entrevista & $\begin{array}{c}\text { Formación académica y } \\
\text { profesional }\end{array}$ & 6 entrenadores (regional) \\
\hline $\begin{array}{c}\text { Modolo, Madeira, Santos, } \\
\text { D’Almeida y Menezes (2017) }\end{array}$ & Cualitativo & Entrevista & Contextos de aprendizaje & 11 entrenadores (escuelas) \\
\hline $\begin{array}{l}\text { Pulido, Sanchez-Oliva, San- } \\
\text { chez-Miguel, Leo \& Garcia- } \\
\text { Calvo (2016) }\end{array}$ & Cuantitativo & Cuestionario & $\begin{array}{l}\text { Formación académica del } \\
\text { entrenador y nivel de motiva- } \\
\text { ción del jugador }\end{array}$ & $\begin{array}{l}432 \text { jugadores ( } 10 \text { a } 16 \\
\text { ańos)/ } 88 \text { entrenadores }\end{array}$ \\
\hline Fischer et al. (2016) & Cuantitativo & $\begin{array}{c}\text { Prueba de programa de } \\
\text { computadora }\end{array}$ & Rendimiento de habilidades & $\begin{array}{l}72 \text { entrenadores con certifi- } \\
\text { cación federativa }\end{array}$ \\
\hline Çelik y Yalçinkaya (2015) & Cuantitativo & Cuestionario & Carisma del entrenador & 242 jugadores (nacional) \\
\hline $\begin{array}{l}\text { Cunha, Estriga y Batista } \\
(2014)\end{array}$ & Cualitativo & Entrevista & $\begin{array}{c}\text { Fuentes de conocimiento más } \\
\text { utilizadas }\end{array}$ & 10 entrenadores (nacional) \\
\hline $\begin{array}{l}\text { Debanne, Angel y Fontayne } \\
(2014)\end{array}$ & Cuantitativo & Análisis de juegos & Utilización de Focus Theory & 30 entrenadores (nacional) \\
\hline Debanne (2014) & Cuantitativo & Monitoreo en juegos & $\begin{array}{c}\text { Técnicas de persuasión de los } \\
\text { árbitros }\end{array}$ & $\begin{array}{c}3 \text { entrenadores profesiona- } \\
\text { les } / 14 \text { partidos }\end{array}$ \\
\hline $\begin{array}{c}\text { Guzmán, Calpe-Gómez, San- } \\
\text { tamaría y Burkhard (2014) }\end{array}$ & Cuantitativo & Grabación de voz & $\begin{array}{c}\text { Comportamiento verbal del } \\
\text { entrenador }\end{array}$ & $\begin{array}{c}21 \text { entrenadores (cadete - } \\
\text { nacional) }\end{array}$ \\
\hline $\begin{array}{l}\text { Meletakos, Bayios, Hatziharis- } \\
\text { tos y Psychountaki (2013) }\end{array}$ & Cualitativo & Cuestionario & $\begin{array}{c}\text { Efecto de la experiencia como } \\
\text { entrenador y deportista }\end{array}$ & 289 entrenadores \\
\hline $\begin{array}{c}\text { Guzmán y Calpe-Gómez } \\
\text { (2012) }\end{array}$ & Cualitativo & Grabación de voz & Comunicación del entrenador & $\begin{array}{c}1 \text { partido (1er. división } \\
\text { nacional española) }\end{array}$ \\
\hline $\begin{array}{c}\text { Molina, Godoy, Calvo, Saiz y } \\
\text { Alonso (2012) }\end{array}$ & Cuantitativo & Cuestionario & $\begin{array}{l}\text { Utilización de las experiencias } \\
\text { de los entrenadores }\end{array}$ & 333 entrenadores (nacional) \\
\hline $\begin{array}{c}\text { Mesquita, Borges, Rosado y } \\
\text { Batista (2012) }\end{array}$ & Cuantitativo & Cuestionario & $\begin{array}{c}\text { Competencia y nivel de } \\
\text { formación }\end{array}$ & $\begin{array}{c}207 \text { entrenadores (16 a } 64 \\
\text { años) }\end{array}$ \\
\hline Debanne y Fontayne (2012) & Cualitativo & Monitoreo en juegos & Comunicación del entrenador & 3 entrenadores (nacional) \\
\hline $\begin{array}{c}\text { Mesquita, Borges, Rosado y } \\
\text { De Souza (2011) }\end{array}$ & Cuantitativo & Cuestionario & $\begin{array}{l}\text { Valor atribuido a la competen- } \\
\text { cia laboral en relación con la } \\
\text { formación }\end{array}$ & $\begin{array}{l}207 \text { entrenadores (16 a } 64 \\
\text { años) }\end{array}$ \\
\hline
\end{tabular}




\begin{tabular}{ccccc}
\hline Autores & Tipo & Método & Variable & Participantes \\
\hline $\begin{array}{c}\text { Molina, Godoy y Delgado } \\
(2010 \mathrm{a})\end{array}$ & Cuantitativo & Cuestionario & $\begin{array}{c}\text { Influencia de la formación en } \\
\text { el desempeño profesional }\end{array}$ & 234 entrenadores (nacional) \\
\hline $\begin{array}{c}\text { Molina, Godoy y Delgado } \\
(2010 \mathrm{~b})\end{array}$ & Cuantitativo & Cuestionario & Nivel de educación & $\begin{array}{c}\text { 269 entrenadores con certifi- } \\
\text { cación federativa }\end{array}$ \\
\hline $\begin{array}{c}\text { Hagemann, Strauss y Büsch } \\
(2008)\end{array}$ & $\begin{array}{c}\text { Cuantitativo } \\
\text { y cualitativo }\end{array}$ & $\begin{array}{c}\text { Resolución de escenarios } \\
\text { yrabación del desempe- } \\
\text { no de los entrenadores }\end{array}$ & $\begin{array}{c}\text { Capacidad de resolución de } \\
\text { problemas }\end{array}$ & 81 entrenadores (nacional) \\
\hline Godoy (2006) & Cualitativo & $\begin{array}{c}\text { Revisión de trabajo } \\
\text { científico }\end{array}$ & $\begin{array}{c}\text { Prácticas profesionales de } \\
\text { entrenadores de balonmano }\end{array}$ \\
\hline
\end{tabular}

\section{Discusión}

El objetivo de este estudio fue realizar una revisión sistemática sobre entrenadores de balonmano para discutir las posibles fronteras del conocimiento sobre este tema en artículos más recientes. A partir de los análisis, fue posible identificar que la producción sobre el tema aún es incipiente, corroborando Prieto et al. (2015), pero con un interés creciente, principalmente alrededor de 2014. Se observó que los artículos analizados se publicaron principalmente por investigadores de universidades europeas y escrito en inglés, similar al panorama científico del balonmano reportado por Aguilar (2014) y Prieto et al. (2015).

En los resultados de este estudio se identificó un aumento en el interés científico en el balonmano desde 2009 corroboran la revisión de Aguilar (2014), mientras que Modolo, Beltramini y Menezes (2018) identificaron un aumento irregular en el interés en los estudios de porteros de balonmano desde 2010.

De los artículos seleccionados, el $64.3 \%$ provienen de países del continente europeo. Estos hallazgos también se pueden observar en estudios como Prieto et al. (2015) y Modolo et al. (2018) que destacan mayor interés de los países europeos en las investigaciones científicas relacionadas con el balonmano, mostrando una posible relación con sus resultados expresivos en competiciones de clase mundial, con la popularidad del balonmano en estos y con el interés de los investigadores en este tema.

Contradictorio a su expresión en el campo competitivo, hubo un gran interés de los investigadores brasileños en el tema (32.1\% de los artículos analizados en este estudio), que puede atribuirse a dos factores: 1) el interés en comprender el desempeńo y la capacitación de entrenadores en balonmano brasileńo por diferentes autores (Modolo et al. al., 2017; Musa et al., 2017); y 2) la inclusión del portugués como idioma de búsqueda de artículos, tal como lo presenta Modolo et al. (2018).

Considerando el lenguaje de las publicaciones, los hallazgos revelaron que el idioma inglés es el más frecuente en relación con el tema investigado, lo que reafirma la perspectiva de Volpato (2011) y corrobora los hallazgos de Aguilar (2014) y Prieto et al. (2015).

Este estudio reveló una visión general de los tipos de investigación más utilizados. Se observó la preferencia por la investigación cualitativa, especialmente con entrevistas para la producción de datos, a diferencia de Modolo et al. (2018) que encontró una predominancia de las investigaciones cuantitativas en su revisión con porteros del balonmano.

En esta revisión se identificó que las investigaciones centradas en el rendimiento fue uno de los más recurrentes con énfasis en las investigaciones centradas en las diferentes etapas de la formación deportiva, lo que revelan la necesidad (y la brecha) de profundizar estos temas, basados en la realidad experimentada por los entrenadores de este contexto (Menezes et al., 2016; Menezes, et al., 2015). Además, se identificó una preocupación por el aprendizaje de los entrenadores, ya que señalan que su formación académica inicial era insuficiente para dominar los contenidos necesarios (Molina, Godoy \& Delgado, 2010a). Este hecho puede estar relacionado con la necesidad del entrenador deportivo se relacione con diferentes contextos y demandas presentadas por el entorno profesional (Cushion, Armor \& Jones, 2003),

Por lo tanto, para proporcionar una capacitación más completa para los entrenadores, se sugiere que se preste mayor atención a las influencias sociales (Mesquita et al., 2012), las experiencias en el entorno profesional y deportivo (Cunha et al., 2014; Feu Molina et al., 2012; Meletakos et al., 2013; Modolo et al., 2017; Musa et al., 2017).

\section{Conclusión}

Este estudio puede estimular nuevas producciones sobre entrenadores de balonmano, además de permitir la comprensión de cómo aprenden los entrenadores y la identificación de puntos relevantes a explorar para que puedan dominar los contenidos necesarios para su desempeño profesional. Cabe señalar que los trabajos analizados se limitan a las bases de datos elegidas y los criterios de selección para este estudio.

En los artículos analizados se concluye que hay una mayor frecuencia de investigaciones relacionadas con los países 
europeos, en la lengua inglesa y a partir de 2019. Los artículos analizados mostraron un mayor interés por parte de los investigadores para identificar los elementos técnico-tácticos y las estrategias más importantes para los entrenadores de balonmano, las formas en que dirigen sus equipos, la comunicación y relación con ellos, además de la importancia de las relaciones sociales en su formación profesional. Por fin, se observó la necesidad de investigaciones centradas en los mismos temas, pero que investigan el contexto de las primeras etapas del entrenamiento deportivo en balonmano y en contextos diferentes a los europeos.

\section{Referencias}

1. Aguilar, O. C. (2014). Análisis de la producción científica en balonmano en las revistas de la Web of Science. E-balonmano.com: Revista de Ciencias del Deporte, 10(2), 77-88.

2. Annerstedt, C. \& Lindgren, E. C. (2014). Caring as an important foundation in coaching for social sustainability: a case study of a successful Swedish coach in high-performance sport. Reflective Practice, 15(1), $27-$ 39.

3. Bormann, K. C., Schulte-Coerne, P., Diebig, M. \& Rowold, J. (2016). Athlete characteristics and team competitive performance as moderators for the relationship between coach transformational leadership and athlete performance. Journal of Sport and Exercise Psychology, 38(3), 268-281.

4. Calpe-Goméz, V., Guzmán, J. F. L. \& Santamaría, C. G. (2013). Relationship between score and coaches' verbal behaviour. Journal of Human Sport and Exercise, 8(3), S728-S737.

5. Cunha, A., Estriga, M. L. D. \& Batista, P. (2014). Fontes de conhecimento percebidas pelos treinadores: estudo com treinadores de handebol da $1^{\text {a }}$ divisão de seniores masculinos em Portugal. Movimento, 20(3), 917-940.

6. Cushion, C. J., Armour, K. M. \& Jones, R. L. (2003). Coach Education and Continuing Professional Development: Experience and Learning to Coach. Quest, 55, 215-230.

7. Dallegrave, E., Mendes, J. \& Nascimento, J. (2018). Estrutura organizativa do processo de treinamento técnico-tático nas categorias de formação no handebol feminino. E-balonmano.com: Revista de Ciencias del Deporte, 14(2), 65-70.

8. Debanne, T. (2014). Techniques used by coaches to influence referees in professional team handball. International Journal of Sports Science and Coaching, 9(3), 433-446.

9. Debanne, T., Angel, V. \& Fontayne, P. (2014). Decision-Making during Games by Professional Handball Coaches Using Regulatory Focus Theory. Journal of Applied Sport Psychology, 26(1), 111-124.

10. Debanne, T. \& Fontayne, P. (2009). A study of a successful experienced elite handball coach's cognitive processes in competition situations. International Journal of Sports Science \& Coaching, 4(1), 1-16.

11. Debanne, T. \& Fontayne, P. (2012). Communication from professional coaches to athletes during handball games according to the "Coaching Model” perspective. Science et Motricite, (76), 11-23.

12. Debanne, T., Fontayne, P. \& Bourbousson, J. (2014). Professional handball coaches management of players' situated understanding during official games. Psychology of Sport and Exercise, 15(6), 596-604.

13. Debanne, T. \& Laffaye, G. (2013). Coaches' beliefs and knowledge: Training programs used by french professional coaches to increase ball-throwing velocity in elite handball players. International Journal of Sports Science and Coaching, 8(3), 557-569.

14. Fernandez-Rios, L. \& Buela-Casal, G. (2009). Standards for the preparation and writing of Psychology review articles. International Journal of Clinical and Health Psychology, 9(2), 329-344.

15. Fischer, L., Baker, J., Rienhoff, R., Strauß, B., Tirp, J., Büsch, D. \& Schorer, J. (2016). Perceptual-cognitive expertise of handball coaches in their young and middle adult years. Journal of Sports Sciences, 34(17), 1637-1642.
16. Galvão, T., Pansani, T. \& Harrad, D. (2015). Principais itens para relatar revisôes sistemáticas e meta-análises: a recomendação PRISMA. Epidemiologia e Serviços de Saúde, 24(2), 335-342.

17. Gama, D., Nunes, R., Castro, J., Souza C., Júnior, F. R. \& Vale, R. (2019). Analysis of the relationship between personality traits and leadership characteristics of handball coaches of school teams in the state of Rio de Janeiro, Brazil. Motriz, 25(3), 1-7.

18. Godoy, S. I. (2006). El Perfil de los entrenadores de balonmano. La formación como factor de cambio. Revista de Ciencias del Deporte, 2(4), 67-68.

19. Gomes, R. \& Paiva, P. (2010). Liderança, compatibilidade treinadoratleta e satisfação no andebol: percepção de atletas novatos e experientes. Psico-USF, 15, 235-248.

20. Gómez-López, M., Martínez, B., Ruiz-Sánches, V. (2018). Orientación de los entrenadores de balonmano. Influencia del género, edad, experiencia, formación y dedicación semanal. E-balonmano.com: Revista de Ciencias del Deporte, 14(1), 27-34.

21. Gómez-López, M., Manzano-Sánchez, D., Merino-Barreno, J \& Valero-Valenzuela, A. (2019). The importance of the coach in predicting implicit beliefs about skills and beliefs about the causes of success in handball players. International Journal of Environmental Research and Public Health, 16, 1-9.

22. Granero-Gallegos, A., Gómez-López, M., Rodríguez-Suárez, N., Abraldes, J. A., Alesi, M. \& Bianco, A. (2017). Importance of the motivacional climate in goal, enjoyment, and the causes of success in handball players. Frontiers of Psychology, 8, 1-8.

23. Guzmán, J. F. \& Calpe-Gómez, V. (2012). Preliminary study of coach verbal behaviour according to game actions. Journal of Human Sport $\mathrm{\sigma}$ Exercise, 7(2), 376-382.

24. Guzmán, J. F. L., Ferriol, A. G. \& Gimeno, E. C. (2005). Percepción de competencia de las jugadoras y de criterios de éxito del entrenador como predictores de la orientación de metas en balonmano de base. Revista de Psicología del Deporte, 14(1), 7-19.

25. Guzmán, J. F. L., Calpe-Gómez, V., Santamaría, C. G. \& Burkhard, F. I. (2014). A systematic observation of coaches' verbal behaviours related to competitive game plays. Revista de Psicología del Deporte, 23(2), 301-307.

26. Hagemann, N., Strauss, B. \& Büsch, D. (2008). The complex problemsolving competence of team coaches. Psychology of Sport and Exercise, 9, 301-317.

27. Hallajy, M., Janani, H. \& Fallah, Z. (2011). Modeling the effect of coaches' leadership styles on athletes' satisfaction and commitment in Iranian handball pro league. World Applied Sciences Journal, 14(9), 1299-1305.

28. Higgins, J. \& Green, S. (2008). Cochrane handbook for systematic reviews of interventions. West Sussex: John Wiley \& Sons Ltd.

29. Hoigaard, R., De Cuyper, B., Fransen, K., Boen, F. \& Peter, D. (2015). Perceived coach behavior in training and competition predicts collective efficacy in female elite handball players. International Journal of Sport Psychology, 46, 321-336.

30. Jones, R. L. \& Hemmestad, L. B. (2019). Reclaiming the 'competent' practicioner: furthering the case for the practically wise coach. Sports Coaching Review, 1-19. 
31. Jones, R. L. \& Thomas, G. L. (2015). Coaching as 'scaffolded' practice: further insights into sport pedagogy. Sports Coaching Review, 4(2), $65-$ 79

32. König, S. (2013). Coaching performance and leadership behaviours in team sports. Sportwissenschaft, 43(4), 253-263.

33. Krahenbühl, T., Galatti, L., Scaglia, A. \& Leonardo, L. (2019). Competição de base e a formação de jovens atletas na perspectiva de treinadores de elite no handebol. Pensar a Prática, 22, 1-13.

34. Krahenbühl, T., Menezes, R. \& Leonardo, L. (2019). Elite coaches' opinion about the additional court player and the strategic-tactical structures in handball. Motriz, 25(3), 1-6.

35. Lee, Y., Kim, S. H. \& Kang, J. H. (2013). Coach leadership effect on elite handball players' psychological empowerment and organizational citizenship behavior. International Journal of Sports Science and Coaching, 8(2), 327-342.

36. Marconi, M. A. \& Lakatos, E. M. (2017). Fundamentos de metodologia cientifica. (8 ed.). São Paulo: Atlas.

37. Marques, R. F. R., Nunomura, M. \& Menezes, R. P. (2016). Sports coaching science in Brazil. Sports Coaching Review, 5(2), 1-6.

38. Meeus, M., Serpa, S. \& De Cuyper, B. (2010). The effects of video feedback on coaches' behavior and the coach-athlete relationship. Journal of Clinic Sport Psychology, 4, 323-340.

39. Meletakos, P., Bayios, I., Hatziharistos, D. \& Psychountaki, M. (2013). Effects of athletic and coaching experience on coaching efficacy in team sports. Gazzetta Medica Italiana Archivio per le Scienze Mediche, 172(6), 457-464.

40. Menezes, R. P. (2018). Ensino do handebol em longo prazo: estudo a partir da opiniāo de treinadores. Educación Física y Ciencia, 20(2).

41. Menezes, R. P., Marques, R. F. R. \& Morato, M. P. (2016). Percepçáo de treinadores de andebol sobre as variáveis defensivas e ofensivas do jogo na categoria sub12. Motricidade, 12(3), 6-19.

42. Menezes, R. P., Marques, R. F. R. \& Nunomura, M. (2015). O ensino do handebol na categoria infantil a partir dos discursos de treinadores experientes. Movimento, 21(2), 463-477.

43. Menezes, R. P., Morato, M. P. \& Reis, H. H. B. (2015). Análise do jogo de handebol na perspectiva de treinadores experientes: categorias de análise ofensivas. Revista da Educação Física/UEM, 26(1), 11-20.

44. Menezes, R. P., Ramos, N. C., Marques, R. F. R. \& Nunomura, M. (2018). Teaching handball to U-16 and U-18 women's teams: coaches' perspective on the long-term. Motriz, 24(4), 1-9.

45. Menezes, R. P. \& Reis, H. H. B. (2017a). Comportamentos ofensivos diante de diferentes cenários defensivos no handebol a partir da opinião de treinadores experientes. Revista da Educação Física, 28(1), 1-14.

46. Menezes, R. P. \& Reis, H. H. B. (2017b). O jogo defensivo diante de diferentes sistemas ofensivos no handebol. análise do cenário técnicotático e reflexões sobre o ensino. Revista Brasileira de Ciências do Esporte, 39(2), 168-175.

47. Menezes, R. P. \& Reis, H. H. B. (2017c). Os discursos dos treinadores de handebol sobre as variáveis individuais e coletivas determinantes para o jogo ofensivo e as possíveis relaçóes com o contexto pedagógico. Revista da Educação Física/UEM, 28(1), e2818.

48. Menezes, R. P. \& Reis, H. H. B. (2014). Relação entre eficácia defensiva e elementos técnico-táticos do handebol a partir dos discursos de treinadores experientes. Revista da Educação Física/UEM, 25(4), 513-526.

49. Mesquita, I., Borges, M., Rosado, A. \& Batista, P. M. (2012). Self-efficacy, perceived training needs and coaching competences: The case of Portuguese handball. European Journal of Sport Science, 12(2), 168-178.

50. Mesquita, I., Borges, M., Rosado, A. \& De Souza, A. D. (2011). Handball coaches' perceptions about the value of working competences according to their coaching background. Journal of Sports Science and Medicine, 10(1), 193-202.

51. Modolo, F., Beltramini, L. \& Menezes, R. (2018). Revisão sistemática sobre o processo de ensino e de análise do goleiro de handebol. Cuadernos de Psicología del Deporte, 18(3), 232-249.

52. Modolo, F., Madeira, M. G., Santos, W. R., D’Almeida, M. d. P. \& Menezes, R. P. (2017). Contextos e situaçôes de aprendizagem de treinadores de handebol em âmbito escolar de um município do Estado de São Paulo. Movimento, 23(4), 1203-1216.

53. Modolo, F. \& Menezes, R. P. (2019). Características técnico-táticas dos goleiros de handebol da categoria sub-16: opinião de treinadores brasileiros. Cuadernos de Psicología del Deporte, 19(1), 206-221.

54. Moesch, K. \& Apitzsch, E. (2012). How do coaches experience psychological momentum? A qualitative study of female elite handball teams. Sport Psychologist, 26(3), 435-453.

55. Moher, D., Liberati, A., Tetzlaff, J \& Altman, D. (2009). Preferred reporting items for Systematic Reviews and Meta-Analysis: the PRISMA statement. Annals of Internal Medicine, 151, 264-270.

56. Molina, S. F., Godoy, S. J. I. \& Delgado, M. G. (2010a). Influencia de la formación formal y no formal en las orientaciones que adoptan los entrenadores deportivos. Revista de Educación, 353, 615-640.

57. Molina, S. F., Godoy, S. J. I. \& Delgado, M. G. (2010b). La formación inicial de los entrenadores de balonmano para la enseńanza del deporte en edad escolar. Cultura, Ciencia y Deporte, 5(14), 109-117.

58. Molina, S. F., Godoy, S. J. I., Calvo, A. L., Saiz, S. J. \& Alonso, M. C. (2012). El conocimiento profesional adquirido por el entrenador de balonmano: experiencias y formación. Revista de Psicología del Deporte, 21(1), 107-115.

59. Molina, S. F., Ibáñez, S. J., Gozalo, M. \& Lorenzo, A. (2010). Decision and planning style of Spanish handball coaches. Open Sports Sciences Journal, 3(1), 111-117.

60. Musa, V., Modolo, F., Barreira, C., Tsuji, G. \& Menezes, R. P. (2017) Representaçóes dos treinadores sobre o papel das instituiçóes reguladoras do handebol para a sua formação. Revista Portuguesa de Ciências do Desporto, S1a, 298-306.

61. Prieto, J., Gómez, M. A. \& Sampaio, J. (2015). A bibliometric review of the scientific production in handball. Cuadernos de Psicología del Deporte, 15(3), 145-154.

62. Pulido, J. J., Sanchez-Oliva, D., Sanchez-Miguel, P. A., Leo, F. M \& Garcia-Calvo, T. (2016). Influencia de la formación de los entrenadores sobre la motivación de los deportistas. Revista Internacional de Medicina y Ciencias de la Actividad Física y del Deporte, 16(64), 685-702.

63. Rivilla-García, J., Sillero, M., Grande, I., Sampedro, J. \& Gómez, M. A. (2014). Do ICT resources improve sports teaching processes in handball? Revista Internacional de Medicina y Ciencias de la Actividad Física y del Deporte, 14(53), 53-67.

64. Santos, W. R. \& Menezes, R. P. (2019). Especialização de jogadoras de handebol a partir dos discursos de treinadores. Cuadernos de Psicología del Deporte, 19(3), 47-61.

65. Silva, A. S., Barreiros, A. \& Fonseca, A. M. (2019). Exploring the importance of coaches in the development of the sports career in handball. The perception of athletes from higher and lower levels of success. Cuadernos de Psicología del Deporte, 19(1), 106-120.

66. Sonoo, C., Hoshino, E. \& Vieira, L. (2008). Liderança esportiva: estudo da percepçâo de atletas e técnicos no contexto competitivo. Psicologia: Teoria e Prática, 10(2), 68-82.

67. Tong, A., Flemming, K., McInnes, E., Oliver, S. \& Craig, J. (2012). Enhancing transparency in reporting the synthesis of qualitative research: ENTREQ. BMC Medical Research Methodology, 12(181), 1-8.

68. Volpato, G. L. (2011). Método lógico para redação científica. Botucatu: Best Writing.

69. Çelik, V. O. \& Yalçinkaya, K. (2015). An examination of charismatic leadership characteristics of handball coaches: perceptions of handball players. Journal of Physical Education \& Sports Science, 9(1), 118-132. 